\title{
THE RATE OF GROWTH OF THE NUMBER OF PRIME ALTERNATING LINKS AND TANGLES
}

\author{
Carl Sundberg and Morwen Thistlethwaite
}

When introduced to the subject of knot theory, it is natural to ask how the number of knots and links grows in relation to crossing number. The purpose of this article is to address this question for the class of prime alternating links; in particular, the exact value of $\lim _{n \rightarrow \infty}\left(A_{n}\right)^{\frac{1}{n}}$ is obtained, where $A_{n}$ is the number of $n$-crossing, prime, unoriented, alternating link types. This result follows from a detailed investigation of the sequence $\left(a_{n}\right)$, where $a_{n}$ is the number of strong equivalence classes of prime, alternating tangle types with $n$ crossings (a tangle equivalence is strong if it fixes the boundary of the ambient ball of the tangle pointwise). The generating function $\sum a_{n} z^{n}$ is shown to satisfy a certain functional equation; a study of the analytic properties of this equation yields an asymptotic formula for $a_{n}$, and a study of its algebraic properties yields a practical method for calculating $a_{n}$ exactly up to several hundred crossings.

\section{Introduction.}

Throughout this paper, the symbol $\sim$ is to be interpreted as follows:

$$
f(n) \sim g(n) \quad \text { means } \quad \lim _{n \rightarrow \infty} \frac{f(n)}{g(n)}=1 .
$$

Theorem 1. Let $a_{n}$ denote the number of prime, alternating tangle types with $n$ crossings. Then

$$
a_{n} \sim \frac{3 c_{1}}{4 \sqrt{\pi}} n^{-\frac{5}{2}} \lambda^{n-\frac{3}{2}}
$$

where

$$
c_{1}=\frac{5^{\frac{7}{2}}}{3^{5} \sqrt{2}} \sqrt{\frac{(21001+371 \sqrt{21001})^{3}}{(17+3 \sqrt{21001})^{5}}}=3.8333138762 \ldots .
$$


and

$$
\lambda=\frac{101+\sqrt{21001}}{40}=6.1479304437 \ldots \ldots
$$

Theorem 2. Let $A_{n}$ denote the number of prime, alternating link types with $n$ crossings. Then

$$
\frac{a_{n-1}}{8(2 n-3)} \leq A_{n} \leq \frac{a_{n-1}}{2}(n \geq 3), \quad \text { and } \quad \lim _{n \rightarrow \infty}\left(A_{n}\right)^{\frac{1}{n}}=\frac{101+\sqrt{21001}}{40} .
$$

\section{Remarks.}

1. The generating function $w=\sum a_{n} z^{n}$ is a root of an irreducible quintic polynomial whose coefficients are polynomials in $z$. The inverse function to $w$ has a radical expression over the polynomial ring $\mathbb{Z}[z]$; this expression was used to compute $a_{n}$ exactly for $n \leq 250$. In conjunction with the first part of Theorem 2, reasonably close bounds are then obtained on the number of alternating link types for $n \leq 251$. Details of the computation of $a_{n}$ are given in $\S 5$.

2. The constant $c_{1}$ is the first order coefficient of a certain Taylor series defined in $\S 4$.

3. It follows immediately from Theorem 1 that $\lim _{n \rightarrow \infty} \frac{a_{n+1}}{a_{n}}=\lambda$.

4. The first proof that the number of $n$-crossing links grows exponentially with increasing $n$ is in $[\mathbf{E S}]$. Also, in $[\mathbf{W}]$, results of $[\mathbf{T u}]$ on the enumeration of planar maps are used to obtain an upper bound on this rate of growth.

Our methods are based on the study of generating functions, and stem from the following sources: (i) J.H. Conway's approach to the enumeration of links, in particular his concepts of "algebraic tangle" and "basic polyhedron" $[\mathrm{C}]$; (ii) Darboux's method for obtaining asymptotic information on the coefficients of a power series, from knowledge of the nature of singularities of the power series; (iii) the solution of the Tait conjecture $[\mathrm{MT}]$; (iv) W. Tutte's determination of the number of rooted c-nets [Tu].

The paper is set out as follows.

$\S 1$. Topological background.

$\S 2$. Enumeration of tangles.

$\S 3$. Proof of Theorem 2.

$\S 4$. Proof of Theorem 1.

$\S 5$. The computation of $a_{n}$.

$\S 6$. Reduced tangle diagrams.

$\S 7$. Non-alternating links. 
We would like to express our thanks to Stefan Richter for insightful conversations, to David Singmaster for directing us to a key paper in the literature, and to Jorge Calvo and Kenneth Millett for helpful comments on a preliminary version of this paper. It is also a pleasure to acknowledge our use of the PARI multiprecision package, written by number theorists C. Batut, D. Bernardi, H. Cohen and M. Olivier, and made freely available by the authors. This package was invaluable in checking results and in computing the values of $a_{n}$ listed in $\S 5$.

\section{Topological background.}

Definition. A tangle is a pair $(B, T)$, where $B$ is the standard 3-ball $\left\{(x, y, z) \in \mathbb{R}^{3}: x^{2}+y^{2}+z^{2} \leq 1\right\}$ and $T$ is a proper, tame 1-submanifold of $B$ meeting $\partial B$ in the four points $P=\left(\frac{1}{\sqrt{2}}, \frac{1}{\sqrt{2}}, 0\right), Q=\left(-\frac{1}{\sqrt{2}}, \frac{1}{\sqrt{2}}, 0\right), R=$ $\left(-\frac{1}{\sqrt{2}},-\frac{1}{\sqrt{2}}, 0\right), S=\left(\frac{1}{\sqrt{2}},-\frac{1}{\sqrt{2}}, 0\right)$, called the ends of $T$.

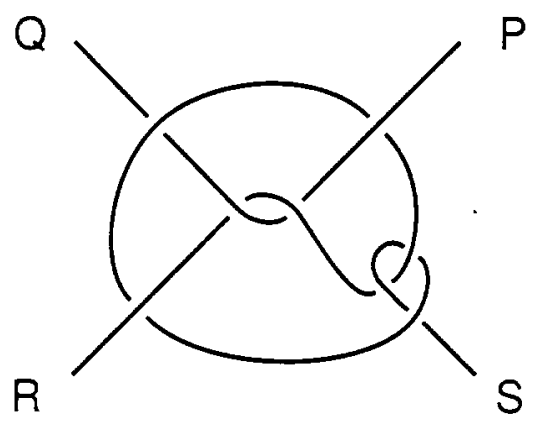

Figure 1.

Let $\Delta$ be the equatorial disk of $B$, i.e. $\Delta=\{(x, y, z) \in B: z=0\}$. We shall take a diagram of a tangle $T$ to be a regular projection of $T$ in $\Delta$, together with an overcrossing-undercrossing structure. As is customary, undercrossings are indicated pictorially by small gaps, as in Figure 1. When we wish to forget the overcrossing-undercrossing structure, we shall refer to the underlying projection of $D$.

Throughout this paper, the term tangle type will signify an equivalence class of tangles under the following strong equivalence relation:

Definition. Tangles $\left(B, T_{1}\right),\left(B, T_{2}\right)$ are equivalent if there exists a homeomorphism $h:\left(B, T_{1}\right) \rightarrow\left(B, T_{2}\right)$, orientation-preserving on $B$, such that $h$ is the identity on $\partial B$.

On occasion, we shall also refer to the following weaker equivalence relation: 
Definition. Tangles $\left(B, T_{1}\right),\left(B, T_{2}\right)$ are weakly equivalent if there exists a homeomorphism $h:\left(B, T_{1}\right) \rightarrow\left(B, T_{2}\right)$ which is orientation-preserving on $B$.

For example, the three tangles illustrated in Fig. 2 are inequivalent, although they are weakly equivalent.
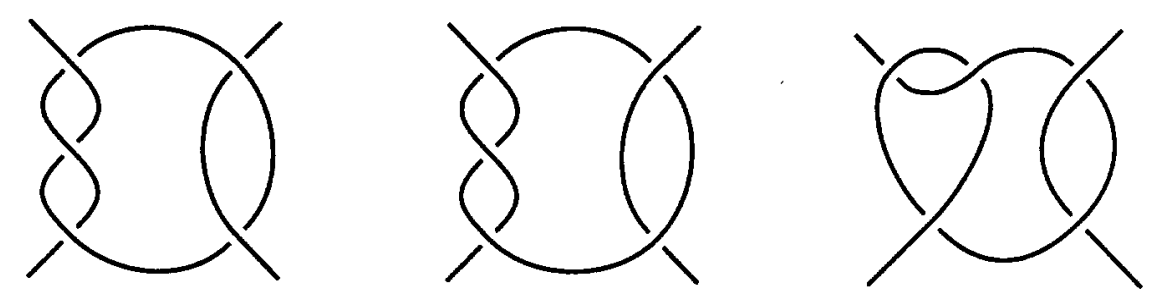

Figure 2.

Definition. A tangle $(B, T)$ is rational if it is weakly equivalent to one of the (trivial) tangles $\left(B, T_{1}\right),\left(B, T_{2}\right)$, where $T_{1}$ consists of the two line segments joining $Q$ to $P, R$ to $S$ respectively, and $T_{2}$ consists of the two line segments joining $Q$ to $R, P$ to $S$ respectively.
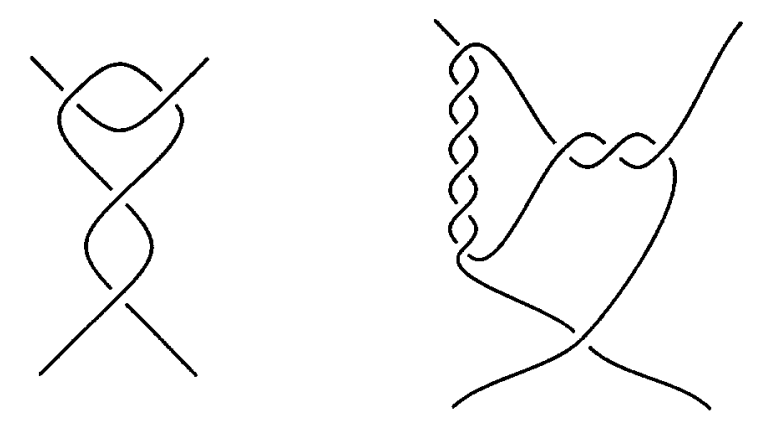

Figure 3. Examples of rational tangles.

As indicated by the title, we are concerned with tangles admitting alternating diagrams. To avoid repetition, we shall assume henceforth that each alternating tangle diagram $D$ satisfies the condition that the edge of $D$ with one end at $P$ has its other end at an overpass. This convention dictates that the edge with one end at $R$ is also incident to an overpass, and that the edges ending at $Q, S$ are incident to underpasses. Moreover, we shall only consider diagrams which are prime according to the following definition.

Definition. A tangle diagram $D$ in a disk $\Delta$ is prime if: 
(i) The underlying projection of $D$ is a connected subset of the disk $\Delta$;

(ii) if $C$ is a circle in the plane meeting $D$ transversely in two points, then these points belong to the same edge of $D$ (i.e. "diagrammatic connected summands" are not allowed).

If a tangle $T$ admits a prime, alternating tangle diagram, the results of $[\mathrm{M}]$ guarantee the following topological constraints on the pair $(B, T)$;

(i) if $S$ is a 2-sphere in $B \backslash T$, then the 3-ball in $B$ bounded by $S$ does not meet $T$;

(ii) if a 2-sphere $S$ in $B$ meets $T$ transversely in two points, then the 3-ball in $B$ bounded by $S$ meets $T$ in an unknotted arc.

From (i), the tangle $T$ cannot be separated by a 2 -sphere in $B$. Also, from (ii), $T$ can be separated by a properly embedded disk in $B$ if and only if $T$ is a rational tangle.

Henceforth, it will be assumed that all tangle diagrams conform to the above requirements.

At this point, we need to consider the circumstances under which two prime, alternating tangle diagrams can represent equivalent tangles.

Let $D$ be a diagram of a tangle $T$, and let $B^{\prime}$ be the closure of the complement in $S^{3}$ of the ball $B$ containing the tangle $T$. We may view $B^{\prime}$ as a rigid vertex attached to the tangle $T$; thus we have associated in a natural way a diagram of a rigid-vertex graph to the original diagram $D$. Since a homeomorphism exhibiting a tangle equivalence fixes pointwise the boundary of $B$, the type of the resulting rigid-vertex graph depends only on the type of the tangle $T$. From the solution of the Tait conjecture [MT], any two alternating diagrams representing the equivalence class of this rigidvertex graph must be related via a sequence of flypes. Hence the same is true for any two alternating diagrams representing the tangle $T$.

Thus we have established that the solution to the Tait flyping conjecture applies equally well to tangle diagrams, though of course the argument of the previous paragraph relied on the fact that equivalences between tangles are of the strong variety. Indeed, it is false that any two alternating diagrams of weakly equivalent tangles are related by a sequence of flypes, as evidenced by the example of Figure 2. The classification of alternating tangles under weak equivalence is feasible, however, and will be undertaken in a separate article.

\section{Enumeration of tangles.}

Let $\left(c_{n}\right)$ be a sequence of real numbers. The generating function for the sequence $\left(c_{n}\right)$ is the power series $\gamma=\sum c_{n} z^{n}$. We may regard $\gamma$ as a formal power series, but often it will be profitable to regard $z$ as a complex 
variable, and $\gamma$ as a function of $z$. We can then invoke techniques of analysis to obtain information on the asymptotic behavior of the coefficients $c_{n}$.

The generating function of primary concern in this paper is

$$
w=\sum a_{n} z^{n},
$$

where $a_{n}$ is the number of $n$-crossing prime, alternating tangle types. The purpose of this section is to establish a functional identity satisfied by $w$. To this end, we shall describe a recursive procedure for constructing alternating tangles, based on an extension of the Conway scheme $[\mathbf{C}]$. The reader familiar with Conway's paper $[\mathbf{C}]$ will recall that Conway generates diagrams of links using two ingredients, namely basic polyhedra and algebraic tangle diagrams. A basic polyhedron is a 4-valent planar map (i.e. graph embedded in $\mathbb{R}^{2}$ ), with no two-sided complementary region. Diagrams are then obtained by substituting algebraic tangle diagrams for each vertex of the basic polyhedron. The power of this method for alternating links of low crossing-number resides in the fact that all flypes occur within the substituent algebraic tangle diagrams. Our approach is essentially the same, but in order to obtain a recursive scheme which is canonical, and which works well with respect to flyping in full generality, we need to refine the definitions slightly.

Definition. A tangle diagram $D$ is the horizontal tangle sum of tangle diagrams $A, B$ if $D$ conforms to Figure $4(\mathrm{i})$, and $D$ is the vertical tangle sum of $A, B$ if $D$ conforms to Figure 4(ii).

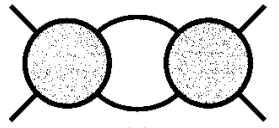

(i)

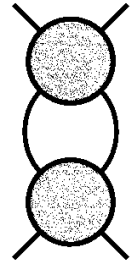

(ii)

Figure 4 .

Recall from $[\mathbf{C}]$ that algebraic tangle diagrams are precisely those obtained recursively from the 1 -crossing diagram $\%$ by means of horizontal and/or vertical tangle sums.

Thus, for example, the tangle diagrams illustrated in Figure 2 are algebraic, and the tangle diagram of Figure 1 is not algebraic. Any tangle which admits an algebraic diagram is algebraic in the sense of [BS]. The converse fails for alternating diagrams, but a characterization of those alternating tangle diagrams which represent algebraic tangles (in the sense of [BS]) is given in $[\mathbf{T h}]$. 
In order to describe a recursive procedure for generating arbitrary tangle diagrams, we shall use the notion of a template, which is a "generalized diagram" obtained from a tangle diagram $D$ by selecting some subset of the set of crossings of $D$, and replacing each crossing from this subset with a small disk called a slot. The motivation behind this is that we can manufacture a tangle diagram $D$ from a template $\tau$ by inserting a tangle diagram into each slot of $\tau$ (see Fig. 5).

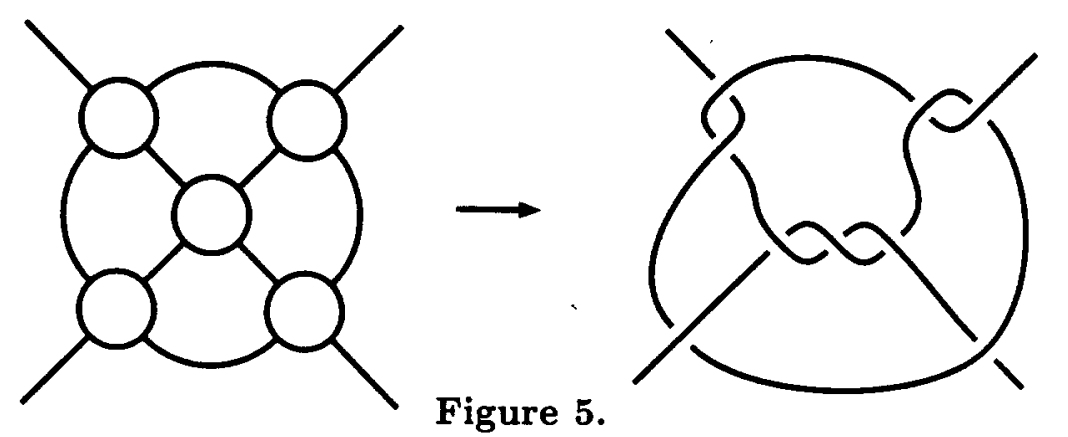

\section{Definition.}

(i) If a template $\tau$ is obtained by replacing some or all of the crossings of a diagram $D$ by slots, we say that $\tau$ is derived from $D$.

(ii) If a tangle diagram $D$ is obtained by inserting tangle diagrams into the slots of a template $\tau$, we say that $D$ is associated with $\tau$.

Note that the concepts "horizontal or vertical tangle sum" and "flype" extend naturally to templates.

We shall need to consider two particular kinds of template.

\section{Definition.}

(i) An algebraic template is a template derived from an algebraic tangle diagram. In particular, an algebraic tangle diagram may be regarded as an algebraic template where the number of slots is zero.

(ii) The algebraic template with one slot and no crossing will be called the trivial algebraic template.

Definition. A basic polyhedral template is a template $\tau$ satisfying the following conditions:

(i) $\tau$ has no crossing;

(ii) $\tau$ has more than one slot;

(iii) $\tau$ contains no "Conway circle", i.e. if a circle in the plane meets $\tau$ transversely in four distinct edges of $\tau$, then either these four edges are all incident to some slot of $\tau$, or else they are incident to the four ends of $\tau$;

(iv) $\tau$ cannot be decomposed as a non-trivial sum of two templates. 


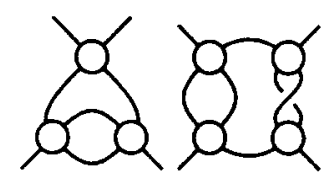

Algebraic templates
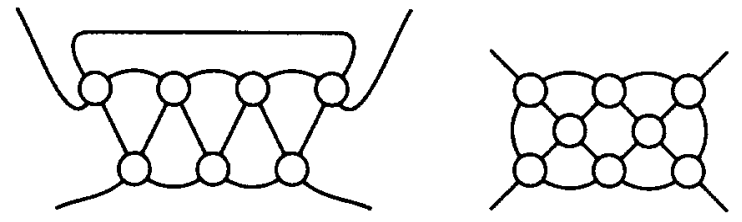

Figure 6.

We digress for a moment to point out an important bijective correspondence between basic polyhedral templates and rooted c-nets, i.e. 3-connected rooted planar maps without multiple joins [Tu]. Given a basic polyhedral template $\tau$ in a disk $\Delta$, in similar spirit to the construction relating to the function $\psi$ in $\S 3$, we may form a 4 -valent planar map $M$ by connecting a vertex in $\mathbb{R}^{2} \backslash \Delta$ to $\tau$ by four arcs, as in Figure 7(i). We regard this extra vertex as being a distinguished vertex of the map $M$. Next, we may shade the regions of $M$ alternately black and white, in checkerboard fashion, so that the region adjacent to the NW and NE ends of $\tau$ are colored black. We can then construct a new planar map $N$ by the medial construction as illustrated in Figure 7(ii); the vertices of $N$ correspond to the white regions of $M$, and the edges of $N$ correspond to the vertices of $M$. In particular, $N$ has one distinguished edge, the root of $N$, passing through the distinguished vertex of $M$. It is easily verified that $N$ is a rooted c-net, and that the assignment of $N$ to $\tau$ is indeed a bijective correspondence between the set of basic polyhedral templates and that of rooted c-nets.

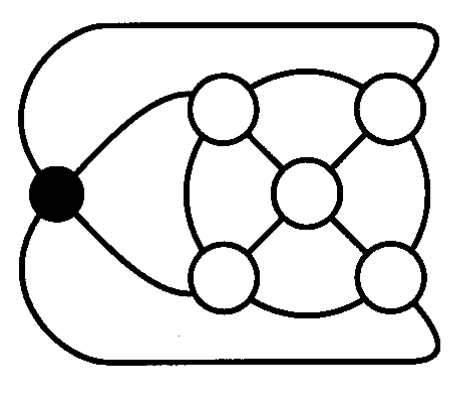

(i)

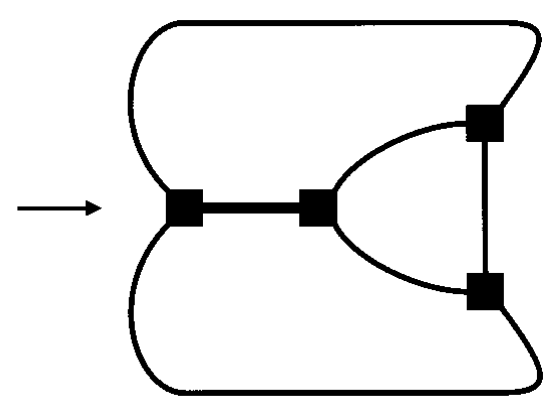

(ii)

Figure 7.

The results of this paper depend in an essential way on Tutte's determination of the rooted c-net generating function, denoted $C(z)$ in [Tu]. Let 
$q$ be the generating function for basic polyhedral templates with $n$ slots. Since a rooted c-net with $n+1$ edges corresponds to a basic polyhedral template with $n$ slots, the functions $q, C$ are related by $z q(z)=C(z)$.

We shall use the following closed formula for $q$, which apparently was not previously known:

$$
q(z)=\frac{1}{2(z+2)^{3}}\left((1-4 z)^{\frac{3}{2}}+\left(2 z^{2}-10 z-1\right)\right)-\frac{2}{1+z}-z+2 .
$$

In [Tu] a recursive formula is given for the number of rooted c-nets with $n$ edges; however, the closed formula (3) may be obtained by algebraic manipulation of expressions in Tutte's paper. On p. 263 of [Tu], on the sixth line after (8.8), the following equations are given:

$$
\eta=-y \frac{(3+\eta)^{2}}{3+2 \eta}, \quad 27 u=-\eta(3+\eta)^{2}
$$

From the first of these equations, $\eta$ can be expressed in terms of $y$ by solving a quadratic equation. If this expression for $\eta$ is substituted into the second equation, $u$ is obtained as a function of $y$. Four lines further down it is stated that $x$ is the same function of $z$ as $u$ is of $y$; therefore the variable $x$ may be eliminated from (8.8), yielding a closed formula for $C(z)$.

Our definition of "basic polyhedral template" corresponds closely to Conway's definition of "basic polyhedron"; apart from the fact that here we are working in the category of tangles rather than links, the only difference is that we have altered the definition slightly so as to exclude Conway circles. Thus, for example, we would not consider the Conway basic polyhedron $10^{* * *}[\mathbf{C}]$ to be "basic", but rather we would consider that it had been obtained by substituting a 5 -crossing tangle into a vertex of $6^{*}$.

We need to single out those tangle diagrams which are associated with basic polyhedral templates.

Definition. A tangle diagram $D$ is of type $\mathrm{I}$ if it is associated with a basic polyhedral template; otherwise it is of type II.

There is a sense in which algebraic and basic polyhedral templates are complementary. It follows from the definitions that any tangle diagram $D$ of type II is associated with a non-trivial algebraic template. This algebraic template might not be unique, as the set of algebraic templates is closed under the operation of substitution; however, $D$ is associated with a unique maximal algebraic template, which is characterized by the property that all substituent tangle diagrams of the template are of type I. 
On the other hand, the absence of Conway circles in a basic polyhedral template implies that any type I tangle is associated with just one basic polyhedral template.

It follows from this discussion that each tangle diagram has a unique "hierarchy", in the sense that there is a unique way of building it recursively from algebraic and basic polyhedral templates, if we impose the single restriction that any substituent of an algebraic template must be of type I. Furthermore, any flype of a tangle diagram arises from a flype of some algebraic template in the hierarchy; therefore, once we have successfully enumerated flype-equivalence classes of algebraic templates, we no longer have to consider flypes in the enumeration of alternating tangle types.

Having defined the recursive procedure for generating tangles, we now turn to enumeration of tangles. The following example illustrates a principle often used in the computation of generating functions.

Let us consider for a moment a basic polyhedral template with $k$ slots, say. Let these slots be labelled $\sigma_{1}, \ldots, \sigma_{k}$, in some arbitrary fixed order. Suppose we wish to manufacture from this template a tangle diagram with $n$ crossings, where $n \geq k$. Thus we insert into each slot $\sigma_{i}$ a tangle diagram with $n_{i}$ crossings, the numbers $n_{i}$ being chosen so that $\sum n_{i}=n$. If $a_{r}$ is the number of tangles with $r$ crossings, then the total number of $n$-crossing tangles obtainable from this template is

$$
\sum a_{n_{1}} a_{n_{2}} \ldots a_{n_{k}}
$$

where the sum is taken over all $k$-tuples $n_{1}, \ldots, n_{k}$ such that $\sum n_{i}=n$. Therefore, if

$$
w=\sum_{r=1}^{\infty} a_{r} z^{r}
$$

is the generating function for the number of alternating tangles, this template yields a contribution to $a_{n}$ which is equal to the coefficient of $z^{n}$ in the formal product $w^{k}$.

Similarly, if $\tau$ is an algebraic template with $k$ slots and $\ell$ free crossings, then the contribution to $a_{n}$ from $\tau$ is $z^{\ell} u^{k}$, where $u$ is the generating function for the number of type I tangles.

Let us use this principle to compute the generating function for flypeequivalence classes of algebraic templates. Since an algebraic template has two attributes, namely the number of crossings and the number of slots, this generating function will be a function of two variables. Specifically, we shall denote the generating function as

$$
\alpha(z, \zeta)=\sum_{m=1}^{\infty} \sum_{n=1}^{\infty} a_{m n} z^{m} \zeta^{n}
$$


where $a_{m n}$ is the number of flype-equivalence classes of algebraic templates with $m$ crossings and $n$ slots.

\section{Proposition 2.1.}

$$
\alpha(z, \zeta)=\frac{1}{2}\left((1+z-\zeta)-\sqrt{(1-z+\zeta)^{2}-\frac{8}{1-z}\left(z^{2}-z \zeta+\zeta\right)}\right) .
$$

Proof. Let $\gamma(z, \zeta)=\sum \sum g_{m n} z^{m} \zeta^{n}$ be the generating function for the set consisting of (i) flype-equivalence classes of algebraic templates which are decomposable as a non-trivial horizontal tangle sum, together with (ii) the trivial algebraic template. Since there is an obvious bijective correspondence between templates decomposable as a horizontal sum and those decomposable as a vertical sum, we have the identity

$$
\alpha(z, \zeta)=2 \gamma(z, \zeta)+z-\zeta
$$

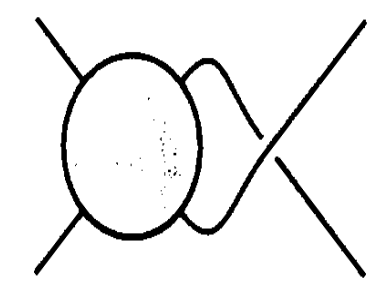

(i)

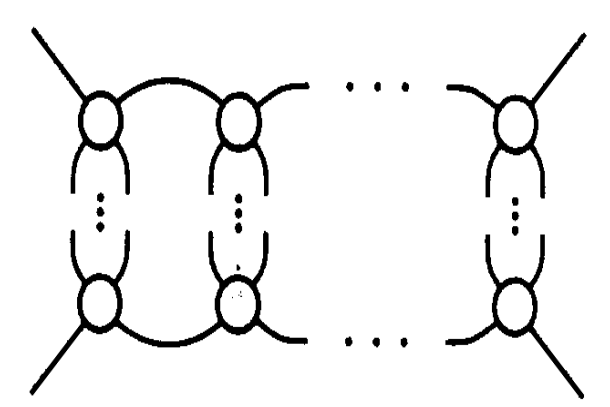

(ii)

Figure 8.

Now consider an algebraic template $\tau$ with $m$ crossings and $n$ slots, decomposable as a horizontal tangle sum. If $\tau$ should have a summand with just one crossing, then by flyping we may assume that this summand is situated at the extreme right of $\tau$ (Fig. 8(i)). Thus $\tau$ is the sum of an arbitrary algebraic template with $m-1$ crossings and $n$ slots with a 1-crossing tangle diagram. Thus the contribution given to $g_{m n}$ by this type of diagram is precisely $a_{m-1, n}$, i.e. the coefficient of $z^{n}$ in $z \alpha=z(2 \gamma+z-\zeta)$. If, on the other hand, $\tau$ does not have a summand with a single crossing, then $\tau$ is a horizontal sum of $k$ tangles where $k$ is some integer $\geq 2$ and each summand is either a vertically decomposable tangle or a trivial 
algebraic template (Fig. 8(ii)). The contribution given to $g_{m n}$ by this type of diagram is equal to the coefficient of $z^{m} \zeta^{n}$ in

$$
\gamma^{2}+\gamma^{3}+\gamma^{4}+\ldots=\frac{\gamma^{2}}{1-\gamma}
$$

Therefore $\gamma$ satisfies the identity

$$
\gamma=z(2 \gamma+z-\zeta)+\frac{\gamma^{2}}{1-\gamma}+\zeta
$$

or

$$
2 \gamma^{2}-(1-z+\zeta) \gamma+\frac{z^{2}}{1-z}+\zeta=0
$$

$\gamma$ is now obtained by application of the quadratic formula, the choice of solution being determined by the condition $\gamma(0,0)=0$. The formula for the generating function $\alpha$ now follows from (5).

Continuing the general discussion, let $\mathcal{T}$ denote the set of all alternating tangle types, and let $\mathcal{U}$ denote the subset of $\mathcal{T}$ consisting of equivalence classes of alternating tangles of type I.

Let $w$ denote the generating function for $\mathcal{T}$, and let $u$ denote the generating function for $\mathcal{U}$.

Since any tangle is obtained by inserting a type I tangle into each slot of an algebraic template, we obtain

$$
w(z)=\alpha(z, u(z))
$$

Also, since any type I tangle is obtained by inserting an arbitrary tangle into the slots of a basic polyhedral template, we obtain

$$
u(z)=q(w(z))
$$

where $q$ is the basic polyhedral template generating function (3). Combining these gives the following functional identity for $w(z)$ :

$$
w(z)=\alpha(z, q(w(z)))
$$

Formulae (3) and (4) yield an explicit expression, involving radicals, for the right-hand side of (6). In $\S 5$ it is explained how to exploit this so as to expand $w$ as a power series in $z$. 


\section{Proof of Theorem 2.}

The main concern of this section is the proof of the first part of Theorem 2; the second part then follows easily, using Theorem 1.

First, we must set up a suitable mapping from the set of $n$-crossing alternating tangles to the set of $(n+1)$-crossing alternating links. In similar spirit to the rigid-vertex construction used in the previous section, we may obtain from each prime, alternating tangle diagram with $n$ crossings a link diagram by adjoining a single crossing, as in Figure 9(i). The resulting $(n+1)$-crossing link diagram will also be prime, and it will be alternating if and only if the correct strand of this extra crossing is chosen to be the overcrossing.

Conversely, we may start with a prime, alternating link diagram $D$ with $n+1$ crossings, and produce an $n$-crossing prime, alternating tangle diagram by selecting any one of the $n+1$ crossings of $D$ and deleting a neighborhood of it. In this process, a further choice is needed, namely a choice of any one of the eight "cyclic" bijections between the set of four edges incident to the crossing and the set of tangle ends $\{P, Q, R, S\}$, corresponding to the elements of the dihedral group of a square. If necessary, we reflect the resulting tangle diagram in the projection plane so as to keep to the convention that the arc with one end at $P$ is incident to an overpass.

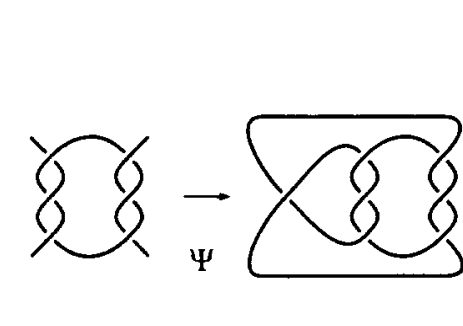

(i)

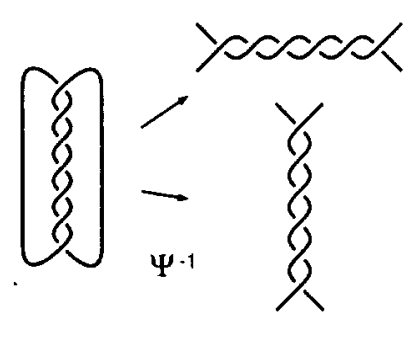

(ii)

\section{Figure 9.}

Since the action of attaching the extra crossing is canonical, this action defines a function, $\psi$ say, from the set of $n$-crossing prime, alternating tangle types to the set of $(n+1)$-crossing prime, alternating link types. We note that $\psi^{-1}(L)$ is small in relation to $n$ if and only if all alternating diagrams of $L$ are highly symmetrical; excluding the Hopf link, the extreme case is that of a $(2, n)$-torus link $(n \geq 3)$, which yields two tangle types under the operation $\psi^{-1}$ (Fig. 9(ii)). Indeed, the Hopf link yields only one tangle type, and we shall exclude the Hopf link from further discussion. 
Our aim is to obtain an upper bound for the cardinality of $\psi^{-1}(L)$. We need to examine the equivalence class of $D$ under flyping, as different diagrams in this equivalence class might yield different sets of tangles. Let $L$ be an alternating link, and let $D$ be an alternating diagram representing $L$.

Definition. A flyping circuit $\mathcal{C}$ of $D$ is a maximal decomposition of $D$ into a number $k \geq 2$ of non-trivial tangle summands $D_{1}, D_{2}, \ldots . ., D_{k}$, interspersed with crossings called active crossings of $\mathcal{C}$, as indicated in Figure 10(i). It is required that a flyping circuit should contain at least one active crossing overall. The number $k$ is called the weight of the flyping circuit $\mathcal{C}$.

Observation. A crossing of $D$ cannot be an active crossing of more than one flyping circuit of $D$.

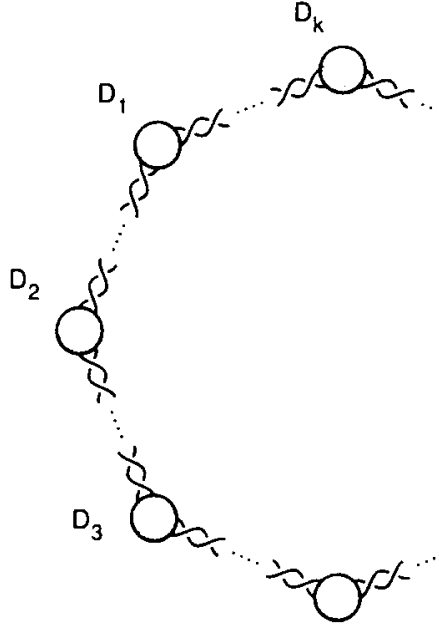

(i)

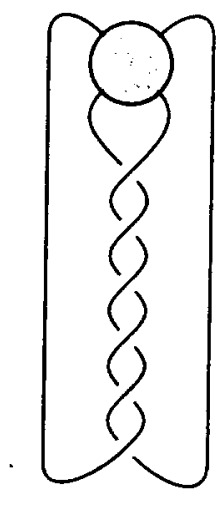

(ii)

Figure 10.

The operation of flyping allows the active crossings to be distributed arbitrarily amongst the "gaps" between the tangle diagrams $D_{i}$, but any flype of $D$ preserves the cyclic arrangement of the $D_{i}$ in the flyping circuit (of course, there may be flypes taking place internally within the $D_{i}$ ). If the gap between $D_{i}$ and $D_{i+1}$ contains active crossings $\nu_{1}, \nu_{2}$, then the tangles obtained from $\nu_{1}, \nu_{2}$ are equivalent. However, if $\nu_{1}, \nu_{2}$ belong to different gaps, then $\nu_{1}, \nu_{2}$ will in general produce inequivalent tangles. If $\nu_{1}, \nu_{2}$ belong to a sequence of crossings as in Fig. 10(ii), corresponding to a degenerate version of a flyping circuit, then none of the crossings in the sequence is 
active, and of course $\nu_{1}, \nu_{2}$ will also produce equivalent tangles. Let us call such a sequence of crossings an inactive twist of $D$. We allow the possibility that an inactive twist might contain just one crossing.

Examples.

(i) The link diagram at the right of Figure 9(i) contains one active crossing (the leftmost crossing), and two inactive twists each with three crossings. The active crossing belongs to a flyping circuit of weight 2 .

(ii) If the function $\psi$ is applied to the tangle diagram of Figure 1, the resulting link diagram has one active crossing (originally adjacent to the end $S$ ), two inactive twists each containing two crossings, and four inactive twists each containing one crossing.

Definition. The weight of a link diagram $D$ is $\gamma(D)=\kappa(D)+\mu(D)$, where $\kappa(D)$ is the sum of the weights of the flyping circuits of $D$, and $\mu(D)$ is the number of inactive twists of $D$.

From the above discussion, we have the following result concerning the function $\psi$ :

Proposition 3.1. Let $L$ be a prime, alternating link type represented by a prime, alternating diagram $D$. Then $\left|\psi^{-1}(L)\right| \leq 8 \gamma(D)$.

The next stage in the proof of Theorem 2 is to obtain an upper bound on $\gamma(D)$. First, we need to extend the notion of a flyping circuit to the case where $D$ is a tangle diagram. Let $\bar{D}$ be the rigid-vertex graph diagram obtained by attaching a 4 -valent rigid vertex to the ends of $D$, as in Fig. 7(i). The concepts of flyping circuit, active crossing and inactive twist extend in an obvious way to rigid-vertex graph diagrams: We simply regard the rigid vertex as a variant of a tangle diagram, but with no internal structure. We now declare that flyping circuits, active crossings, inactive circuits and inactive twists of the tangle diagram $D$ are simply the corresponding attributes of the associated rigid-vertex graph diagram $\bar{D}$.

Proposition 3.2. Let $D$ be a tangle diagram with $n \geq 2$ crossings. Then $\gamma(D) \leq 2 n-3$.

Proof. We proceed by induction on $n$. We first consider two special cases, which constitute the basis for the induction. First, let $D$ be a rational tangle with at least two crossings, with Conway symbol $c_{1} c_{2} \ldots c_{k},[\mathbf{C}]$. Then $D$ is constructed by plaiting in $k$ stages, where $c_{i}$ half-twists are performed at the $k$ th stage. Moreover, $c_{1} \geq 2$, and $c_{i} \geq 1$ for $i \geq 2$. After the first stage, the diagram consists of a single inactive twist, and has weight 1 . At 
each successive stage, a new flyping circuit is created with weight 2 , whereas the status of existing crossings is unaltered. Therefore the weight of $D=$ $c_{1} c_{2} \ldots c_{k}$ is $2 k-1$, whereas the number of crossings of $D$ is $\sum_{i=1}^{k} c_{i} \geq k+1$. Therefore the conclusion holds in this case.

The second special case is that of an $n$-crossing tangle diagram of type I, associated with a basic polyhedral template whose substituents are all single crossings. Then $\kappa(D)=0$ and $\mu(D)=n$, so $\gamma(D)=n$, and since $n \geq 5$ the conclusion holds in this case also.

Now let $D$ be an $n$-crossing tangle diagram not covered by these two special cases. Suppose first that $D$ is of type I, associated with a basic polyhedral template with $k$ slots. Let this template have $m \geq 1$ substituent tangle diagrams $D_{1}, D_{2}, \ldots, D_{m}$, with more than one crossing, and $k-m$ single-crossing substituents. Let $D_{i}$ have $n_{i}$ crossings $(1 \leq i \leq m)$, and let $D_{i}$ have weight $w_{i}$. Then the weight of $D$ is

$$
\gamma(D)=\sum_{i=1}^{m} w_{i}+(k-m) \leq \sum_{i=1}^{m}\left(2 n_{i}-3\right)+(k-m)=2 \sum_{i=1}^{m} n_{i}+k-4 m
$$

and the crossing-number of $D$ is $n=\sum_{i=1}^{m} n_{i}+(k-m)$. But

$$
(2 n-3)-\gamma(D) \geq k+2 m-3>0, \text { since } k \geq 5 .
$$

Finally, suppose that $D$ is of type II. Then $D$ is a non-trivial tangle sum of $k$ tangles, say, where $k \geq 2$. Since we have already dealt with the case where $D$ consists of a single twist, we may assume that at least one of the summands has more than one crossing. Let the non-trivial summands be $D_{1}, D_{2}, \ldots, D_{m}$, where $m \geq 1$. If $k>m$, then these tangles belong to a flyping circuit of $D$ of weight $m+1$, and with $k-m$ active crossings. Again, let $D_{i}$ have crossing-number $n_{i}$ and weight $w_{i},(1 \leq i \leq m)$. Then the number of crossings of $D$ is

$$
n=\sum_{i=1}^{m} n_{i}+(k-m)
$$

and the weight of $D$ is

$$
\gamma(D)= \begin{cases}\sum_{i=1}^{m} w_{i}+m+1 & (k>m) \\ \sum_{i=1}^{m} w_{i} & (k=m)\end{cases}
$$

By induction,

$$
\gamma(D) \leq \sum_{i=1}^{m}\left(2 n_{i}-3\right)+m+1=\sum_{i=1}^{m} 2 n_{i}-2 m+1,
$$


and it follows that

$$
(2 n-3)-\gamma(D) \geq 2 k-4 \geq 0 .
$$

Remark. It follows from the proof of Proposition 3.2 that the upper bound $2 n-3$ is achieved only for rational tangles of form $2111 \ldots 1$.

Proposition 3.3. Let $D$ be a prime, alternating link diagram with $n \geq 3$ crossings. Then $\gamma(D) \leq 2 n-3$.

Proof. If $D$ should have no flyping circuit, then $\gamma(D)$ is equal to the total number of inactive twists of $D$, whence $\gamma(D) \leq n$. Therefore the conclusion follows in this case.

Suppose that $D$ has a flyping circuit with $k \geq 2$ tangle diagrams $D_{1}$, $D_{2}, \ldots, D_{k}$ and $\alpha$ active crossings. Let $D_{i}$ have crossing-number $n_{i}$ and weight $w_{i}$. From Lemma 3.1, $w_{i} \leq 2 n_{i}-3$. Therefore

$$
\gamma(D)=\sum_{i=1}^{k} w_{i}+k \leq 2 \sum_{i=1}^{k} n_{i}-2 k .
$$

Therefore

$$
(2 n-3)-\gamma(D) \geq(2 \alpha-3)+2 k>0 .
$$

Corollary 3.3.1. Let $D$ be a prime, alternating link diagram with $n \geq 3$ crossings. Then $2 \leq\left|\psi^{-1}(L)\right| \leq 8(2 n-3)$.

Remark. The upper bound of Corollary 3.3.1 is a little conservative; but the aim is to show that there exists an upper bound which is linear in $n$.

The first part of Theorem 2, namely

$$
\frac{a_{n-1}}{8(2 n-3)} \leq A_{n} \leq \frac{a_{n-1}}{2}
$$

follows at once from Corollary 3.3.1. Taking logarithms and dividing by $n$, we obtain

$$
\frac{\log \left(a_{n-1}\right)}{n}-\frac{\log (8(2 n-3))}{n} \leq \frac{\log \left(A_{n}\right)}{n} \leq \frac{\log \left(a_{n-1}\right)}{n}-\frac{\log (2)}{n} .
$$

By Theorem 1, the outermost expressions of this inequality both tend to $\log (\lambda)$ for large $n$ so we have proved that

$$
\lim _{n \rightarrow \infty} \frac{\log \left(A_{n}\right)}{n}=\log (\lambda), \quad \text { or } \quad \lim _{n \rightarrow \infty}\left(A_{n}\right)^{\frac{1}{n}}=\lambda,
$$

thus concluding the proof of Theorem 2 . 


\section{Proof of Theorem 1.}

In this section we establish the asymptotic formula of Theorem 1 . The generating function $w$ is defined as the formal power series $\sum a_{n} z^{n}$, where $a_{n}$ is the number of prime, alternating tangle types with $n$ crossings; however, if we are to apply analytical techniques directly to $w$, we need to verify that the series $\sum a_{n} z^{n}$ has a positive radius of convergence.

Proposition 4.1 ([Tu, W]). The radius of convergence of $w$ is at least $\frac{4}{27}$.

Proof. We may associate bijectively to each tangle diagram a rooted nonseparable planar map, by means of the medial construction explained in $\S 2$. Therefore the radius of convergence of $w$ cannot be less than that of the generating function $B(x)$ for rooted non-separable planar maps with $n$ edges. But from the formula for $B(x)$ ([Tu], 6.4), the radius of convergence of $B(x)$ is $\frac{4}{27}$. (See also [W] for a discussion of closely related matters.)

Proposition 4.1 has the following interpretation. If we did not impose the equivalence relation of flyping on alternating tangles, the radius of convergence of the generating function $w$ would be precisely $\frac{4}{27}$. Incorporating flypes evidently reduces the number of objects being counted, and the amount by which the radius of convergence of $w$ exceeds $\frac{4}{27}$ is in some sense a measure of this reduction.

Recall that the function $w$ satisfies the functional identity (6), which we restate in the following convenient form:

$$
F(z, w)=w(1+z)-w^{2}-(w+1) q(w)-z-\frac{2 z^{2}}{1-z}=0 .
$$

We now introduce a number fundamental to this paper. Referring to (7), the equation $F\left(z, \frac{1}{4}\right)=0$, after multiplication by $1-z$, becomes a quadratic equation in $z$. It is readily verified that this equation has a positive root, which we shall denote $r_{0}$. From (3), $q\left(\frac{1}{4}\right)=\frac{1}{540}$, so we have

$$
r_{0}=\frac{\sqrt{21001}-101}{270} .
$$

The next task is to prove that $r_{0}$ is the radius of convergence of the series $\sum a_{n} z^{n}$. It is necessary to proceed in careful steps, as initially we do not know whether $w(z)$ is represented by a power series at any point outside the circle $|z|=\frac{4}{27}$. First, we show that there is a unique function $w:\left[0, r_{0}\right] \rightarrow\left[0, \frac{1}{4}\right]$ of class $\mathcal{C}^{1}$ satisfying (7) together with the condition 
$w(0)=0$. Since for the time being we are concerned only with real numbers, we introduce

$$
u=\Re w, \quad x=\Re z,
$$

and re-write the identity (7) as

$$
F(x, u)=u(1+x)-u^{2}-(u+1) q(u)-x-\frac{2 x^{2}}{1-x}=0 .
$$

Let $D$ denote the rectangle $\left\{(x, u) \subseteq \mathbb{R}^{2}: 0 \leq x \leq r_{0}\right.$ and $\left.0 \leq u \leq \frac{1}{4}\right\}$. Since the function $q$ is of class $\mathcal{C}^{1}$ on $\left[0, \frac{1}{4}\right]$, it follows that $F$ is of class $\mathcal{C}^{1}$ on $D$.

In the following lemma, the symbols $\frac{\partial F}{\partial u}, \frac{\partial F}{\partial x}$ refer to one-sided derivatives where appropriate.

Lemma 4.2.1. (i) $\frac{\partial F}{\partial u}>0$ on $D$, and (ii) $\frac{\partial F}{\partial x}<0$ on $D$.

Proof. Since the functions $q, q^{\prime}$ are represented by series with non-negative coefficients on $\left[0, \frac{1}{4}\right]$, both $q, q^{\prime}$ are increasing on $\left[0, \frac{1}{4}\right]$. We have

$$
\begin{aligned}
\frac{\partial F}{\partial u} & =(1+x)-\left(2 u+q(u)+(u+1) q^{\prime}(u)\right) \\
& >1-\left(\frac{1}{2}+q\left(\frac{1}{4}\right)+\frac{5}{4} q^{\prime}\left(\frac{1}{4}\right)\right) \\
& =\frac{1}{2}-\frac{1}{540}-\frac{5}{4} \cdot \frac{167}{2025} \\
& >0
\end{aligned}
$$

Also, we have

$$
\frac{\partial F}{\partial x}<u-1<0
$$

Lemma 4.2.2. For each $x \in\left[0, r_{0}\right]$, there exists precisely one number $u \in\left[0, \frac{1}{4}\right]$ such that $F(x, u)=0$.

Proof. First we show that if $x \in\left[0, r_{0}\right], F(x, u)=0$ has at least one solution for $u \in\left[0, \frac{1}{4}\right]$. From (9) it is readily checked that $F(x, 0) \leq 0$ and that $F\left(x, \frac{1}{4}\right) \geq 0$, for $x \in\left[0, r_{0}\right]$. By continuity it follows that for each $x \in\left[0, r_{0}\right], F(x, u)=0$ for some $u \in\left[0, \frac{1}{4}\right]$.

To show that there cannot be more than one solution, it is sufficient to observe that Lemma 4.2.1 guarantees that $F(x, u)$ is an increasing function of $u$ for $0 \leq u \leq \frac{1}{4}$. 
It follows from Lemmas 4.2.1, 4.2.2 that we have defined a function from $\left[0, r_{0}\right]$ to $\left[0, \frac{1}{4}\right]$, which is represented by the power series $\sum a_{n} x^{n}$ within the interval of convergence of that series. Therefore it is appropriate to denote this function $u:\left[0, r_{0}\right] \rightarrow\left[0, \frac{1}{4}\right]$. Since $\frac{\partial F}{\partial u}$ exists and is non-zero on $D$, the derivative of $u$ exists on $\left[0, r_{0}\right]$. Also, since $\frac{\partial F}{\partial u}, \frac{\partial F}{\partial x}$ have opposite signs on $D$, the derivative of $u$ is strictly positive on $\left[0, r_{0}\right]$.

Returning now to the complex variables $z, w$, it is clear that the function $F$ in the identity $(7)$ is analytic at points $(z, w) \in \mathbb{R}^{2}$ belonging to the interior of $D$. Therefore we may use the complex version of the Implicit Function Theorem, together with Lemma 4.2.1, to deduce that there exists a neighborhood $N$ of the half-open interval $\left[0, r_{0}\right)$ in the complex plane, and a function $\phi$ defined on $N$, with the following properties:

(i) $\phi$ agrees with $u$ on $\left[0, r_{0}\right)$, and

(ii) $\phi$ is analytic on $N$.

We are now in a position to prove:

\section{Proposition 4.2.}

(i) The radius of convergence of $w$ is $r_{0}$.

(ii) The series $\sum a_{n} r_{0}^{n}$ converges to $w\left(r_{0}\right)=\frac{1}{4}$.

Proof. (i) Let the radius of convergence of $w$ be $r$. Since the coefficients of the series $\sum a_{n} z^{n}$ are positive for all sufficiently large $n$, Pringsheim's Theorem $([\mathbf{H}]$, Theorem 5.7.1) ensures that $w$ is singular at $r$. Since we have just shown that $w$ is analytic on the half-open interval $\left[0, r_{0}\right)$, it follows that $r \geq r_{0}$.

To complete the proof of (i), it is sufficient to show that $w$ is singular at $r_{0}$. Let us suppose, to the contrary, that $w$ were analytic at $z=r_{0}$. Then from (6) the composite function $q \circ w$ would be analytic at $z=r_{0}$. But, from Lemma 4.2.1 above, $w^{\prime}\left(r_{0}\right)$ is non-zero; therefore the function $w$ would have an inverse, analytic within a neighborhood of $w\left(r_{0}\right)=\frac{1}{4}$. It would follow that the function $q$ was analytic at $\frac{1}{4}$, contradicting the presence of $(1-4 z)^{\frac{3}{2}}$ in $(3)$.

(ii) It follows from the discussion preceding this proposition that $w$ is continuous at $r_{0}$ from the left. Since $a_{n} \geq 0$ for all $n, w(r)=\sum_{n=0}^{\infty} a_{n} r^{n} \nearrow$ $w\left(r_{0}\right)=\frac{1}{4}$ as $r \nearrow r_{0}$, and it follows that $\sum_{n=0}^{\infty} a_{n} r_{0}^{n} \geq \frac{1}{4}$. Suppose that $\sum_{n=0}^{\infty} a_{n} r_{0}^{n}>\frac{1}{4}$. Then there would exist an $N$ such that $\sum_{n=0}^{N} a_{n} r_{0}^{n}>\frac{1}{4}$, which would mean that for $r<r_{0}$ sufficiently close to $r_{0}, \sum_{n=0}^{N} a_{n} r^{n}>\frac{1}{4}$. This would lead to the inequality $w(r)=\sum_{n=0}^{\infty} a_{n} r^{n} \geq \sum_{n=0}^{N} a_{n} r^{n}>\frac{1}{4}$, a contradiction.

It follows from part (ii) of Proposition 4.2 that for any point $z$ on the 
circle $|z|=r_{0}$ the series $\sum a_{n} z^{n}$ is absolutely convergent, hence convergent. Therefore the series $\sum a_{n} z^{n}$ represents $w$ as a continuous function on the closed disk $|z| \leq r_{0}$.

Moreover, since there is at least one tangle of any given crossing-number, the series $w(z)=\sum a_{n} z^{n}$ has two adjacent terms with strictly positive coefficients. For non-real $z$, the corresponding terms in the series $w\left(z_{0}\right)=$ $\sum a_{n} z_{0}^{n}$ will be complex numbers with differing arguments, and so $\left|\sum_{n=0}^{\infty} a_{n} z^{n}\right|<\sum_{n=0}^{\infty} a_{n}|z|^{n}$. Therefore, for all points in the closed disk $|z| \leq r_{0}$ other than $r_{0}$, we have the strict inequality $|w(z)|<w\left(r_{0}\right)=\frac{1}{4}$.

The next proposition elucidates the behavior of $w(z)$ on its circle of convergence.

Proposition 4.3. $\quad w$ is analytic at all points other than $r_{0}$ on the circle $|z|=r_{0}$.

Proof. With a view to using the Implicit Function Theorem, we first show that $\frac{\partial F}{\partial w}$ cannot vanish at $(z, w(z))$ when $z$ is in the closed disk $|z| \leq r_{0}$. In the third inequality we make repeated use of the fact that for a series $\sum k_{n} z^{n}$ with $k_{n} \geq 0,\left|\sum k_{n} z^{n}\right| \leq \sum k_{n}|z|^{n}$; also, in the fourth inequality we use the fact that if $0 \leq z \leq r_{0}$, then $0 \leq w(z) \leq w\left(r_{0}\right)=\frac{1}{4}$.

We have

$$
\begin{aligned}
\left|\frac{\partial F}{\partial w}(z, w(z))\right| & =\left|1+z-\left(2 w(z)+q(w(z))+(w(z)+1) q^{\prime}(w(z))\right)\right| \\
& \geq|1+z|-\left|2 w(z)+q(w(z))+(w(z)+1) q^{\prime}(w(z))\right| \\
& \geq|1+z|-\left(|2 w(z)|+|q(w(z))|+\left|(w(z)+1) q^{\prime}(w(z))\right|\right) \\
& \geq|1+z|-\left(2 w(|z|)+q(w(|z|))+(w(|z|)+1) q^{\prime}(w(|z|))\right) \\
& \geq\left(1-r_{0}\right)-\left(2 w\left(r_{0}\right)+q\left(w\left(r_{0}\right)\right)+\left(w\left(r_{0}\right)+1\right) q^{\prime}\left(w\left(r_{0}\right)\right)\right) \\
& =\frac{1}{2}-\frac{1}{540}-\frac{5}{4} \frac{167}{2025}-r_{0} \\
& >0
\end{aligned}
$$

We have already seen that $w\left(r_{0}\right)=\frac{1}{4}$, and that $\left|w\left(z_{0}\right)\right|<\frac{1}{4}$ for points $z_{0}$ such that $\left|z_{0}\right|=r_{0}, z_{0} \neq r_{0}$. Now the function $q(w)$ is analytic for $|w|<\frac{1}{4}$. Therefore the function $F(z, w)$ is analytic at points $(z, w(z))$ for which $z$ is a point on the circle $|z|=r_{0}$ distinct from $r_{0}$. The conclusion now follows by application of the Implicit Function Theorem.

Remark. Once we have established the asymptotic formula of Theorem 1 , it will be evident that the "growth factor" $\lambda$ of Theorems 1, 2 is simply the reciprocal of $r_{0}$. 
Let us now introduce variables

$$
\zeta=(1-4 w)^{\frac{1}{2}}, \quad \eta=\left(r_{0}-z\right)^{\frac{1}{2}} .
$$

Our immediate aim is to show that $\zeta$ is an analytic function of $\eta$ within a neighborhood of $z=r_{0}$.

We define functions $f, g$ as follows:

$$
f(w)=\frac{1}{2(w+2)^{3}}, \quad g(w)=\frac{2 w^{2}-10 w-1}{2(w+2)^{3}}-\frac{2}{1+w}-w+2 .
$$

Then $f, g$ are analytic at $w=\frac{1}{4}$, and from (3)

$$
q(w)=\zeta^{3} f(w)+g(w) .
$$

Also, we note that

$$
g\left(\frac{1}{4}\right)=q\left(\frac{1}{4}\right)=\frac{1}{540}, \quad g^{\prime}\left(\frac{1}{4}\right)=q^{\prime}\left(\frac{1}{4}\right)=\frac{167}{2025} .
$$

The functional equation (7) may now be written

$$
\frac{\left(1-\zeta^{2}\right)^{2}}{16}-\frac{1-\zeta^{2}}{4}+\frac{\zeta^{2}}{4} z+\frac{5-\zeta^{2}}{4}\left(\zeta^{3} f(w)+g(w)\right)=-\frac{3}{4} z-\frac{2 z^{2}}{1-z},
$$

where for conciseness we have written $w$ for $\frac{1-\zeta^{2}}{4}$. The value of the left-hand side of (11) at $w=\frac{1}{4}, z=r_{0}$ is

$$
\frac{1}{16}-\frac{1}{4}+\frac{5}{4} g\left(\frac{1}{4}\right)=-\frac{5}{27} .
$$

Subtracting this quantity from each side of (11), we obtain

$$
\zeta^{2} U(\zeta, z)=\left(r_{0}-z\right) V(z),
$$

where

$$
\begin{gathered}
U(\zeta, z)=\frac{1}{8}+\frac{z}{4}+\frac{\zeta^{2}}{16}+\frac{\zeta\left(5-\zeta^{2}\right)}{4} f(w)-\frac{1}{4} g(w)+\frac{5}{4} \frac{g(w)-g\left(\frac{1}{4}\right)}{\zeta^{2}} \\
V(z)=\frac{5}{4(1-z)}\left(\frac{101+\sqrt{21001}}{270}+z\right) .
\end{gathered}
$$


Now

$$
\begin{aligned}
U\left(0, r_{0}\right) & =\frac{1}{8}+\frac{r_{0}}{4}-\frac{1}{4} q\left(\frac{1}{4}\right)+\frac{5}{16} q^{\prime}\left(\frac{1}{4}\right) \\
& =\frac{r_{0}}{4}+\frac{8}{81}=\frac{3 \sqrt{21001}+17}{3240}, \\
V\left(r_{0}\right) & =\frac{5}{4\left(1-r_{0}\right)} \frac{\sqrt{21001}}{135}=\frac{21001+371 \sqrt{21001}}{46656} .
\end{aligned}
$$

Each of these expressions is non-zero. Therefore there exist functions $G(\zeta, z), H(z)$, analytic within a neighborhood of $\zeta=0, z=r_{0}$, such that

$$
G^{2}=U, \quad H^{2}=V
$$

Recalling from (10) that $\eta=\left(r_{0}-z\right)^{\frac{1}{2}}$, we now have

$$
\zeta G\left(\zeta, r_{0}-\eta^{2}\right)=\eta H\left(r_{0}-\eta^{2}\right),
$$

which will define $\zeta$ as an analytic function of $\eta$ within a neighborhood of $\eta=0$ by the Implicit Function Theorem, provided the derivative of the left-hand side of (14) with respect to $\zeta$ is non-zero at $\zeta=\eta=0$. But the value of this derivative at $\zeta=\eta=0$ is simply $G\left(0, r_{0}\right)$, which we have just seen is non-zero. Since $\zeta=0$ when $\eta=0$, we have therefore proved

Proposition 4.4. Let $\zeta=(1-4 w)^{\frac{1}{2}}, \eta=\left(r_{0}-z\right)^{\frac{1}{2}}$. Then there exists a neighborhood $N$ of $\eta=0$ and a function $\Phi(\eta)$ analytic on $N$, such that

$$
\zeta=\eta \Phi(\eta) \quad(\eta \in N) .
$$

We now list some assorted facts linking derivatives of $\zeta$ with derivatives of $\Phi$ and the functions $G, H$. They may all be obtained by straightforward differentiation.

$$
\begin{gathered}
\frac{\partial G}{\partial \zeta}\left(0, r_{0}\right)=-\frac{5}{8} f\left(\frac{1}{4}\right)\left(G\left(0, r_{0}\right)\right)^{-1}=-\frac{20}{729}\left(G\left(0, r_{0}\right)\right)^{-1} \\
\left.\frac{d \zeta}{d \eta}\right|_{\eta=0}=\frac{H\left(r_{0}\right)}{G\left(0, r_{0}\right)}=\Phi(0) \\
\left.\frac{d^{2} \zeta}{d \eta^{2}}\right|_{\eta=0}=-2 \frac{\left(H\left(r_{0}\right)\right)^{2}}{\left(G\left(0, r_{0}\right)\right)^{3}} \frac{\partial G}{\partial \zeta}\left(0, r_{0}\right)=2 \Phi^{\prime}(0) .
\end{gathered}
$$


Our overall aim is to obtain asymptotic information on the coefficients $a_{n}$ of the power series $w=\sum a_{n} z^{n}$. To this end, we shall use the recently gleaned information on the function $\Phi(\eta)$. From (15) together with $w=$ $\frac{1}{4}\left(1-\zeta^{2}\right)$, we obtain

$$
w=\frac{1}{4}-\frac{1}{4}\left(r_{0}-z\right)(\Phi(\eta))^{2}
$$

Let us write

$$
-\frac{1}{4}(\Phi(\eta))^{2}=\sum_{0}^{\infty} c_{n} \eta^{n}
$$

Then, using (12), (13), (16)-(18),

$$
\begin{aligned}
c_{0} & =-\frac{1}{4}(\Phi(0))^{2}, \\
c_{1} & =-\frac{1}{2} \Phi(0) \Phi^{\prime}(0)=\frac{10}{729} \sqrt{\frac{V\left(r_{0}\right)^{3}}{U\left(0, r_{0}\right)^{5}}} \\
& =\frac{5^{\frac{7}{2}}}{3^{5} \sqrt{2}} \sqrt{\frac{(21001+371 \sqrt{21001})^{3}}{(17+3 \sqrt{21001})^{5}}} .
\end{aligned}
$$

We note that both $c_{0}, c_{1}$ are non-zero.

We are finally in a position to extract the asymptotic information we desire regarding the coefficients $a_{n}$ of $w$. From (19), (20) we have

$$
w=\frac{1}{4}-\frac{1}{4}\left(r_{0}-z\right)(\Phi(0))^{2}+\sum_{n=1}^{\infty} c_{n}\left(r_{0}-z\right)^{1+\frac{n}{2}} .
$$

Subtracting the first term of the sum from both sides of this equation gives

$$
w-c_{1}\left(r_{0}-z\right)^{\frac{3}{2}}=\frac{1}{4}-\frac{1}{4}\left(r_{0}-z\right)(\Phi(0))^{2}+\sum_{n=2}^{\infty} c_{n}\left(r_{0}-z\right)^{1+\frac{n}{2}} .
$$

We now observe that the third derivative with respect to $z$ of the right-hand side of (22) contains just one term of negative exponent, namely $c_{3}\left(r_{0}-z\right)^{-\frac{1}{2}}$; therefore this third derivative is integrable on its circle of convergence. Let

$$
\psi(z)=\frac{d^{3}}{d z^{3}}\left(w-c_{1}\left(r_{0}-z\right)^{\frac{3}{2}}\right)=\sum u_{n} z^{n} \text { say }
$$

where the power series expansion is valid for $|z| \leq r_{0}, z \neq r_{0}$. The key to obtaining the asymptotic estimate for $a_{n}$ will be: 
Proposition 4.5. $\lim _{n \rightarrow \infty} u_{n} r_{0}^{n}=0$.

The proof of Proposition 4.5 uses standard analytic techniques; a detailed account of the proof is given, as it is anticipated that most readers will not be specialists in the field of analysis. We begin with a lemma.

\section{Lemma 4.5.1.}

$$
\lim _{r \nearrow r_{0}} \int_{-\pi}^{\pi}\left|\psi\left(r_{0} e^{i \theta}\right)-\psi\left(r e^{i \theta}\right)\right| \frac{d \theta}{2 \pi}=0
$$

Proof. We may write

$$
\psi(z)=\frac{h(z)}{\left(r_{0}-z\right)^{\frac{1}{2}}}+k(z)
$$

where $h, k$ are analytic in a neighborhood of $r_{0}$. We choose $\delta>0$ such that $h, k$ are analytic within a neighborhood of the $\operatorname{arc}\left\{r_{0} e^{i \theta}:-\delta<\theta<\delta\right\}$.

Since $\psi$ is continuous at all points of the circle $|z|=r_{0}$ other than $r_{0}$, it is sufficient to prove that

$$
\lim _{r \nearrow r_{0}} \int_{-\delta}^{\delta}\left|\psi\left(r_{0} e^{i \theta}\right)-\psi\left(r e^{i \theta}\right)\right| \frac{d \theta}{2 \pi}=0 .
$$

Also, since it is clear from continuity that

$$
\int_{-\delta}^{\delta}\left|k\left(r_{0} e^{i \theta}\right)-k\left(r e^{i \theta}\right)\right| \frac{d \theta}{2 \pi} \rightarrow 0 \quad \text { as } r \nearrow r_{0}
$$

all we need show is that

$$
\lim _{r \succ r_{0}} \int_{-\delta}^{\delta}\left|\frac{h\left(r_{0} e^{i \theta}\right)}{\left(r_{0}-r_{0} e^{i \theta}\right)^{\frac{1}{2}}}-\frac{h\left(r e^{i \theta}\right)}{\left(r_{0}-r e^{i \theta}\right)^{\frac{1}{2}}}\right| \frac{d \theta}{2 \pi}=0 .
$$

We split up the integral (24) as $I_{1}+I_{2}$, where

$$
\begin{aligned}
& I_{1}=\int_{|\theta|<\eta}\left|\frac{h\left(r_{0} e^{i \theta}\right)}{\left(r_{0}-r_{0} e^{i \theta}\right)^{\frac{1}{2}}}-\frac{h\left(r e^{i \theta}\right)}{\left(r_{0}-r e^{i \theta}\right)^{\frac{1}{2}}}\right| \frac{d \theta}{2 \pi}, \\
& I_{2}=\int_{\eta<|\theta|<\delta}\left|\frac{h\left(r_{0} e^{i \theta}\right)}{\left(r_{0}-r_{0} e^{i \theta}\right)^{\frac{1}{2}}}-\frac{h\left(r e^{i \theta}\right)}{\left(r_{0}-r e^{i \theta}\right)^{\frac{1}{2}}}\right| \frac{d \theta}{2 \pi},
\end{aligned}
$$

$\eta$ being some small number to be chosen presently. 
By continuity, there exists $M$ such that $|h(z)|<M$ for all $z$ in a neighborhood of the arc $\left\{r_{0} e^{i \theta}:-\delta<\theta<\delta\right\}$. Then, for all $r$ sufficiently close to $r_{0}$ and all sufficiently small $\eta$,

$$
\begin{aligned}
& \int_{-\eta}^{\eta}\left|\frac{h\left(r_{0} e^{i \theta}\right)}{\left(r_{0}-r_{0} e^{i \theta}\right)^{\frac{1}{2}}}-\frac{h\left(r e^{i \theta}\right)}{\left(r_{0}-r e^{i \theta}\right)^{\frac{1}{2}}}\right| \frac{d \theta}{2 \pi} \\
& <\frac{M}{r_{0}^{1 / 2}} \int_{-\eta}^{\eta} \frac{d \theta /(2 \pi)}{\left|1-e^{i \theta}\right|^{\frac{1}{2}}}+\frac{M}{r^{1 / 2}} \int_{-\eta}^{\eta} \frac{d \theta /(2 \pi)}{\left|\left(r_{0} / r\right)-e^{i \theta}\right|^{\frac{1}{2}}} \\
& <\frac{M}{r_{0}^{1 / 2}} \int_{-\eta}^{\eta} \frac{d \theta /(2 \pi)}{\left|1-e^{i \theta}\right|^{\frac{1}{2}}}+\frac{M}{r^{1 / 2}} \int_{-\eta}^{\eta} \frac{d \theta /(2 \pi)}{\mid 1-e^{\left.i \theta\right|^{\frac{1}{2}}}} \\
& <\frac{3 M}{r_{0}^{1 / 2}} \int_{-\eta}^{\eta} \frac{d \theta /(2 \pi)}{\left|1-e^{i \theta}\right|^{\frac{1}{2}}}<\frac{4 M}{r_{0}^{1 / 2}} \int_{-\eta}^{\eta} \frac{d \theta /(2 \pi)}{|\theta|^{\frac{1}{2}}} .
\end{aligned}
$$

Given $\epsilon>0$, we can choose $\eta$ such that the last integral of (25) is less than $\frac{\epsilon}{2}$. For this $\eta$, we may choose $r_{1}$ such that $r_{1}<r<r_{0}$ implies that $I_{2}<\frac{\epsilon}{2}$, on account of the fact that

$$
\frac{h\left(r e^{i \theta}\right)}{\left(r_{0}-r e^{i \theta}\right)^{1 / 2}} \rightarrow \frac{h\left(r_{0} e^{i \theta}\right)}{\left(r_{0}-r_{0} e^{i \theta}\right)^{1 / 2}} \quad \text { uniformly for } \eta<|\theta|<\delta .
$$

Thus if $r_{1}<r<r_{0}$, we see that

$$
\int_{-\delta}^{\delta}\left|\frac{h\left(r_{0} e^{i \theta}\right)}{\left(r_{0}-r_{0} e^{i \theta}\right)^{\frac{1}{2}}}-\frac{h\left(r e^{i \theta}\right)}{\left(r_{0}-r e^{i \theta}\right)^{\frac{1}{2}}}\right| \frac{d \theta}{2 \pi} \leq I_{1}+I_{2}<\epsilon .
$$

Proof of Proposition 4.5.

For $0<r<r_{0}$, we have

$$
\int_{-\pi}^{\pi} \psi\left(r e^{i \theta}\right) e^{-i n \theta} \frac{d \theta}{2 \pi}=u_{n} r^{n}
$$

and it follows from Lemma 4.5.1 that

$$
u_{n} r_{0}^{n}=\int_{-\pi}^{\pi} \psi\left(r_{0} e^{i \theta}\right) e^{-i n \theta} \frac{d \theta}{2 \pi}
$$

Let $\epsilon>0$. By Lemma 4.5.1 again, we may choose $0<r<r_{0}$ such that

$$
\int_{-\pi}^{\pi}\left|\psi\left(r_{0} e^{i \theta}\right)-\psi\left(r e^{i \theta}\right)\right| \frac{d \theta}{2 \pi}<\frac{\epsilon}{2} .
$$


Also, since $\sum u_{n} r^{n}$ converges for $0<r<r_{0}$, we may choose $N$ such that $n \geq N$ implies that $\left|u_{n} r^{n}\right|<\frac{\epsilon}{2}$. Then, from (26), (27),

$$
\begin{aligned}
\left|u_{n} r_{0}^{n}\right| & =\left|u_{n}\left(r_{0}^{n}-r^{n}\right)\right|+\left|u_{n} r^{n}\right| \\
& \leq \int_{-\pi}^{\pi}\left|\psi\left(r_{0} e^{i \theta}\right)-\psi\left(r e^{i \theta}\right)\right| \frac{d \theta}{2 \pi}+\left|u_{n} r^{n}\right|<\epsilon .
\end{aligned}
$$

We now conclude the proof of Theorem 1. From (22) we have

$$
\begin{aligned}
u_{n} r_{0}^{n} & =n(n+1)(n+2) a_{n+3} r_{0}^{n}-\frac{3}{8} c_{1} r_{0}^{-\frac{3}{2}} \frac{3}{2} \cdot \frac{5}{2} \cdot \frac{7}{2} \ldots \ldots \cdot \frac{2 n+1}{2} \frac{1}{n !} \\
& =n(n+1)(n+2) a_{n+3} r_{0}^{n}-\frac{3}{4 \sqrt{\pi}} c_{1} r_{0}^{-\frac{3}{2}} \frac{\Gamma\left(n+\frac{3}{2}\right)}{\Gamma(n+1)} .
\end{aligned}
$$

Since the second term of this difference has a positive lower bound, we deduce that

$$
n(n+1)(n+2) a_{n+3} r_{0}^{n} \sim \frac{3}{4 \sqrt{\pi}} c_{1} r_{0}^{-\frac{3}{2}} \frac{\Gamma\left(n+\frac{3}{2}\right)}{\Gamma(n+1)} .
$$

But from Stirling's formula,

$$
\lim _{n \rightarrow \infty} \frac{\Gamma\left(n+\frac{3}{2}\right)}{\Gamma(n+1)}=\sqrt{n}
$$

so we deduce that

$$
n^{3} a_{n+3} r_{0}^{n} \sim \frac{3}{4 \sqrt{\pi}} c_{1} n^{\frac{1}{2}} r_{0}^{-\frac{3}{2}}
$$

and finally that

$$
a_{n} \sim \frac{3}{4 \sqrt{\pi}} c_{1} n^{-\frac{5}{2}} r_{0}^{-\left(n-\frac{3}{2}\right)}
$$

\section{The Computation of $a_{n}$.}

From the functional equation (7) and the closed formula (3) for $q$, one can easily obtain an explicit formula involving radicals for $z$ in terms of $w$. From this formula, a program such as PARI will produce a series for $z$ in powers of $w$, to a specified number of terms, from which the series for $w$ can be obtained by series inversion. By this method $a_{n}$ was computed on 
a PC for $n \leq 250$; the entire computation took about $1 \frac{1}{2}$ minutes. Table 1 lists $a_{n}$ for $n \leq 50$.

\begin{tabular}{||l|l||l|l||}
\hline \hline$n$ & $a_{n}$ & $n$ & $a_{n}$ \\
\hline 1 & 1 & 26 & 10509472317890690 \\
2 & 2 & 27 & 58659056351295672 \\
3 & 4 & 28 & 328591560659948828 \\
4 & 10 & 29 & 1846850410940949702 \\
5 & 29 & 30 & 10412612510292744992 \\
6 & 98 & 31 & 58877494436409193754 \\
7 & 372 & 32 & 333824674188182988872 \\
8 & 1538 & 33 & 1897547736517167483401 \\
9 & 6755 & 34 & 10811965262963697918390 \\
10 & 30996 & 35 & 61743712191685678016610 \\
11 & 146982 & 36 & 353346524877622075891224 \\
12 & 715120 & 37 & 2026177347462171700453095 \\
13 & 3552254 & 38 & 11640600587816460301163560 \\
14 & 17951322 & 39 & 66996349258043741043340070 \\
15 & 92045058 & 40 & 386246530925962967964742478 \\
16 & 477882876 & 41 & 2230378992391449594561463197 \\
17 & 2508122859 & 42 & 12899088068723013463347397454 \\
18 & 13289437362 & 43 & 74709139668891399374031990962 \\
19 & 71010166670 & 44 & 433304503640281884536963608542 \\
20 & 382291606570 & 45 & 2516458655255640459944907578072 \\
21 & 2072025828101 & 46 & 14633147958143711975783070855666 \\
22 & 11298920776704 & 47 & 85194787578604567988225302223590 \\
23 & 61954857579594 & 48 & 496584378798958350593234750705656 \\
24 & 341427364138880 & 49 & 2897722141068904014462120608500022 \\
25 & 1890257328958788 & 50 & 16927166534580561781535347570516714 \\
\hline \hline
\end{tabular}

Table 1.

It is interesting to compare expected and actual values of $a_{n}$. Substituting $n=50$ into the asymptotic estimate of Theorem 1 yields approximately $1.644 \times 10^{34}$, an error of approximately $3 \%$. For $n=250$, the error is approximately $0.57 \%$.

One may eliminate radicals to obtain $w$ as a root of a polynomial of degree 5 in $w$ and degree 4 in $z$ :

$$
\begin{aligned}
& \left(z^{4}-2 z^{3}+z^{2}\right) w^{5} \\
& +\left(8 z^{4}-14 z^{3}+8 z^{2}-2 z\right) w^{4} \\
& +\left(25 z^{4}-16 z^{3}-14 z^{2}+8 z+1\right) w^{3} \\
& +\left(38 z^{4}+15 z^{3}-30 z^{2}-z+2\right) w^{2} \\
& +\left(28 z^{4}+36 z^{3}-5 z^{2}-12 z+1\right) w \\
& +\left(8 z^{4}+17 z^{3}+8 z^{2}-z\right)
\end{aligned}
$$


For certain integer values of $z$, this yields a quintic polynomial in $w$ whose Galois group is the symmetric group of degree 5; therefore one cannot hope to obtain $w$ as a radical expression in $z$.

\section{Reduced alternating tangles.}

A tangle diagram $D$ is said to be reduced if the ends of $D$ are incident to four distinct crossings of $D$. For example, the diagrams of Figures 1,2 are reduced, whereas a standard diagram of a rational tangle is not reduced (see Figure 3). An $n$-crossing diagram of a rational tangle can be obtained by applying a plait to a 1-crossing tangle diagram; at each stage of the plaiting process one adds a crossing by twisting two adjacent ends of the tangle. Taking flyping into account, at each stage there are essentially two choices involved: One can either twist the NE and SE ends, or one can twist the NE and NW ends (Figure 11). Similarly, any unreduced tangle diagram which is not a rational tangle diagram may be obtained by applying a plait to a unique reduced tangle diagram.
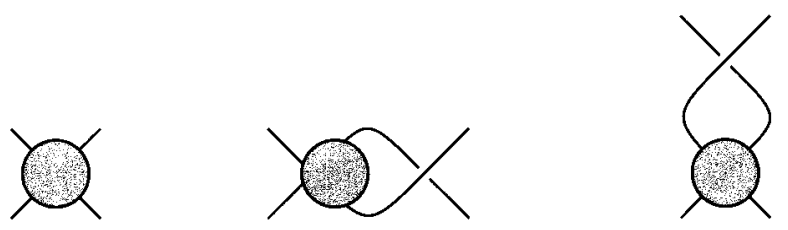

Figure 11.

Let $w_{0}=\sum b_{n} z^{n}$ be the generating function for reduced alternating tangles with $n$ crossings. From the above discussion, we have

$$
w=\left(w_{0}+z\right)\left(1+2 z+(2 z)^{2}+\ldots . .\right)=\frac{1}{1-2 z}\left(w_{0}+z\right)
$$

whence

$$
w_{0}=(1-2 z) w-z .
$$

It follows that

$$
b_{1}=0, \quad b_{n}=a_{n}-2 a_{n-1} \quad(n \geq 2),
$$

and that

$$
b_{n} \sim\left(1-\frac{2}{\lambda}\right) \frac{3 c_{1}}{4 \sqrt{\pi}} n^{-\frac{5}{2}} \lambda^{n-\frac{3}{2}},
$$

where $c_{1}, \lambda$ are as in Theorem 1 . 


\section{Non-alternating links and tangles.}

The machinery developed in this paper can be used to show that the ratio of the number of prime, alternating links with $n$ crossings to the total number of prime links with $n$ crossings tends to zero exponentially. The method is to incorporate a suitable non-alternating tangle into the recursive process for building tangles. The functional equation is then modified slightly, and the resulting generating function has a slightly smaller radius of convergence than $r_{0}$. One has to show that flyping suffices to generate equivalences of tangles built this way; details will appear in a separate article.

\section{References}

[BS] F.Bonahon and L.C. Siebenmann, The classification of arborescent links, preprint.

[C] J.H. Conway, An enumeration of knots and links, Computational Problems in Abstract Algebra, ed. Leech, Pergamon Press 1970, 329-358.

[ES] C. Ernst and D.W. Sumners, The growth of the number of prime knots, Proc. Camb. Phil. Soc., 102 (1987), 303-315.

[H] E. Hille, Analytic function theory, Vol. 1, Ginn and Co., 1962.

[M] W.W. Menasco, Closed incompressible surfaces in alternating knot and link complements, Topology, 23(3) (1984), 37-44.

[MT] W.W. Menasco and M.B. Thistlethwaite, The classification of alternating links, Ann. of Math., 138 (1993), 113-171.

[Th] M.B. Thistlethwaite, On the algebraic part of an alternating link, Pacific J. Math., 151 (1991), 317-333.

[Tu] W. Tutte, A census of planar maps, Canad. J. Math., 15 (1963), 249-271.

[W] D.J.A. Welsh, On the number of knots and links, Colloquia Mathematica Societatis Janos Bolyai, 60 (1991), 713-718.

Received June 13, 1996. The research of the first author was partially supported by NSF Grant no. DMS 9401027 and the research of the second author was partially supported by NSF Grant no. DMS 9401139.

University of Tennessee

KNOXVILLE, TN 37996

E-mail addresses: sundberg@novell.math.utk.edu morwen@math.utk.edu 